\title{
Atomic-scale Feedback-controlled Electron Beam Fabrication of 2D Materials
}

Matthew Boebinger ${ }^{1}$, Sudhajit Misra ${ }^{2}$, Yiling Yu${ }^{1}$, Kai Xiao ${ }^{1}$, Tyler Mathis ${ }^{3}$, Yury Gogotsi ${ }^{3}$, Andrew R. Lupini $^{1}$, Sergei Kalinin ${ }^{4}$, Stephen Jesse ${ }^{5}$ and Raymond Unocic ${ }^{1}$

${ }^{1}$ Oak Ridge National Laboratory, United States, ${ }^{2}$ Oak Ridge National Laboratory, Oakridge, Tennessee, United States, ${ }^{3}$ Drexel University, United States, ${ }^{4}$ Center for Nanophase Materials Sciences, Oak Ridge National Laboratory, Oak Ridge, Tennessee, United States, ${ }^{5}$ Center for Nanophase Materials Sciences, Oak Ridge National Laboratory, United States

A central goal in nanotechnology is to control how nanoscale architectures are fabricated from the level of atoms and up. Recently, scanning transmission electron microscopy (STEM) has been used to precisely manipulate the atomic structure of materials in a way that is impossible to achieve using conventional topdown processing methods [1-3]. This is achieved by controlling the position of a sub- $\AA$ sized electron (e)beam to initiate knock-on processes, thereby enabling the precise atomic alteration of materials. Previous studies demonstrated the ability to selectively position dopant atoms in 2D and 3D materials [1, 3, 4] and to form specific atomic edge configurations or nanopores via controlled e-beam irradiation [5, 6]. Dopants, nanopores, nanowires, and their associated edge structures, have a large effect on the electronic properties of 2D materials that are advantageous for nanoelectronic devices [1-8]; however, the atomistic reaction pathways incurred during the manipulation processes are not well understood. In this study, we demonstrate more precise control of the e-beam for the nanostructure fabrication of 2D materials by using a feedback-controlled e-beam system [7]. This system is used to move the e-beam in a programmed spiral motion at a predetermined dwell time and electron dose, which permits highly accurate control over the location, size, and shape of the desired architectures through automated feedback control. The spiral scans are recorded during atomic fabrication and allows for in situ atomic-resolution STEM imaging of the specific pathways individual atoms move along during fabrication.

For this study a NionUltraSTEM was interfaced with the feedback-controlled e-beam system for automated in situ fabrication of $2 \mathrm{D} \mathrm{Ti}_{3} \mathrm{C}_{2} \mathrm{~T}_{\mathrm{x}} \mathrm{MXene}$, where $\mathrm{T}_{\mathrm{x}}$ represents the surface functional group (-F, $\left.-\mathrm{OH},-\mathrm{O}\right)$, and $\mathrm{MoS}_{2}$ at $100 \mathrm{kV}$ and $60 \mathrm{kV}$, respectively. After each spiral scan (smaller panels, Figure 1), the average detector intensity over the scan area is determined, recorded, and used as a feedback signal. The spiral scan is repeated until the intensity falls below a pre-determined threshold indicating a hole has formed. The degree of control and imaging capabilities allows for tracking individual atoms during the entire atomic manipulation process, which can reveal new insights into the transformation pathways. Figure 1 demonstrates nanopore milling in single layer MXene. The temperature was ramped from $25^{\circ} \mathrm{C}$ to $700^{\circ} \mathrm{C}$ using a Protochips Fusion heater chip to remove the native functional groups that exist on the sample surface [8]. The effect of temperature on the drilling process is shown in the associated spiral scan images (to right of each panel). Atom tracking during drilling reveals the reaction pathways that Ti undergoes as a function of both temperature and MXene surface chemistry. Edge reconstruction and beam irradiation can be tracked and monitored to better control the final structure.

To fabricate atomically thin, metallic nanowires in $\mathrm{MoS}_{2}$, two parallel segments (Figure 2a) were e-beam irradiated. The feedback provided the precise control needed to form the nanowire structure. The e-beam was then rastered across the structure (Fig. 2c-d) to facilitate the formation of other nanostructures - a thin, Morich nanowire forms as observed previously [5,6]. The feedback-controlled system increases the repeatability and reliability in forming these types of nanostructures using e-beam irradiation. This system allows for indepth studies of transformations that high-energy e-beam irradiation induces on 2D materials while also increasing the degree of control available to fabricate nanoscale devices using automated STEM techniques [9]. 

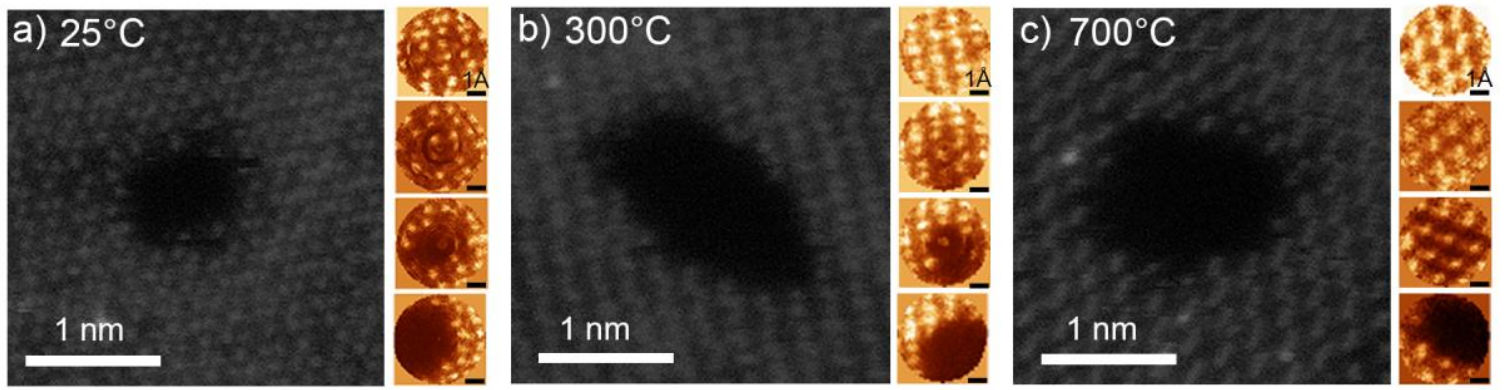

Figure 1. Controlled fabrication of nanopores in MXene as a function of temperature: (a-c) Result of $100 \mathrm{kV}$ e-beam drilling as a function of temperature (a) $25^{\circ} \mathrm{C}$, (b) $300^{\circ} \mathrm{C}$, and (c) $700^{\circ} \mathrm{C}$. Panels on right of each image show several examples of atomically resolved spiral scans acquired during drilling process.

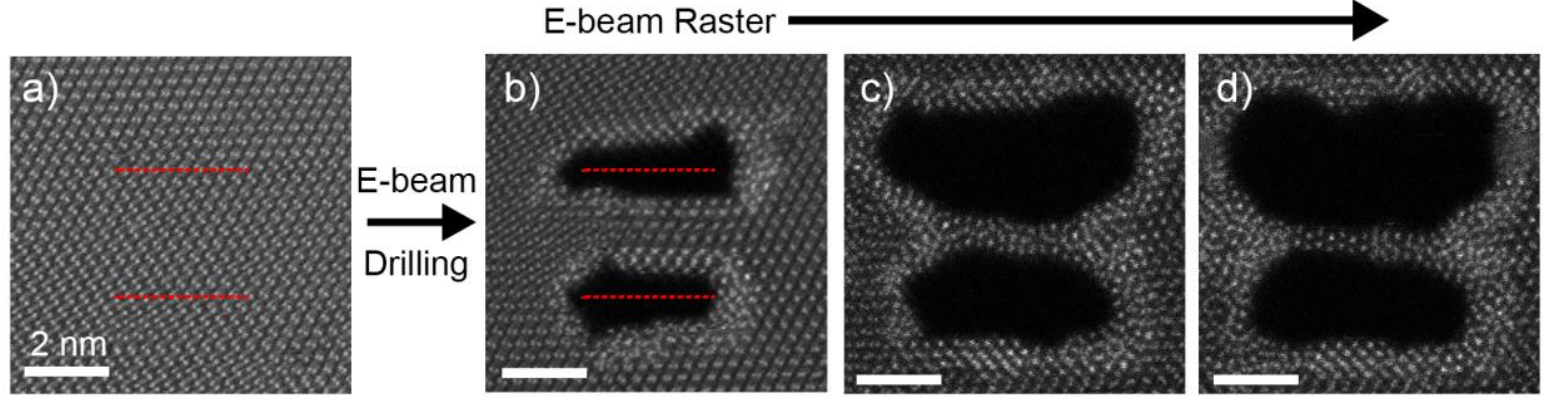

Figure 2. Fabrication of metallic nanowires: (a) Annular dark field STEM image of multi-layer $\mathrm{MoS}_{2}$ prior to atomic fabrication along defined red lines. (b) After drilling a nanowire structure is made, which was found to be repeatable. (c-d) After nanowire formation, e-beam was rastered across viewing area creating different nanowire and edge structures as e-beam continues interacting with sample.

\section{References}

[1] Dyck, O., et al., Nature Reviews Materials 4 (2019) p. 497

[2] Kalinin, S.V., A.Y. Borisevich, and S. Jesse, Nature 539 (2016) p. 485

[3] Tripathi, M., et al., Nano Lett 18 (2018) p. 5319

[4] B. M. Hudak et al. ACS Nano 12 (2018) p. 5873

[5] Sang, X., et al., Nat Commun9 (2018) p. 2051

[6] Lin, J., et al., Nat Nanotechnol9 (2014) p. 436

[7] Jesse, S., et al., Nanotechnology 29 (2018) p. 255303

[8] Hart, J.L., et al., Nat Commun10 (2019) p. 522

[9] Work supported by Oak Ridge National Laboratory's Center for Nanophase Materials Sciences, which is a Department of Energy (DOE), Office of Science User Facility. MXene synthesis was supported by the Fluid Interface Reactions, Structures, and Transport (FIRST) Center, an Energy Frontier Research Center (EFRC) funded by the DOE, Office of Science, Office of Basic Energy Sciences. 